\title{
Design of leaky-wave antennas with transverse slots for end-fire radiation with optimized radiation efficiency
}

\author{
Thomas Vaupel and Claudius Löcker \\ Fraunhofer Institute for High Frequency Physics and Radar Techniques, Fraunhofer Str. 20, 53343 Wachtberg, Germany
}

Correspondence: Thomas Vaupel (thomas.vaupel@ fhr.fraunhofer.de)

Received: 19 February 2019 - Revised: 3 May 2019 - Accepted: 8 May 2019 - Published: 19 September 2019

\begin{abstract}
A substrate integrated waveguide (SIW) with transverse slots on the top plane can be used to design an effective leaky-wave antenna with good frequency beamscanning and platform integration capability. For a main beam near end-fire, the phase constant of the radiating wave must be near to the free space wavenumber or slightly larger. In this context, the modified Hansen-Woodyard condition gives an optimum phase constant to maximize the directivity at end-fire. For the analysis of the wave propagation we have implemented a modal analysis for rectangular waveguides with transverse slots. Near end-fire, three types of modal solutions exists, a leaky improper mode, a surface wave mode and a proper waveguide mode. The leaky mode can reach phase constants larger than the free space wavenumber to fulfill the Hansen-Woodyard condition, but loses strongly its physical significance in this slow wave region, thus the excitation of the leaky-wave becomes negligible there, whereas the proper waveguide mode is dominant but exhibits only a negligible radiation loss leading to a strong drop of the antenna efficiency. Therefore, the optimum efficiency of $86 \%$ for maximizing the gain as proposed in the literature cannot be reached with this kind of leaky wave antenna.

But it will be shown in this contribution by analyzing antenna structures with finite aperture lengths, that the efficiency can reach nearly $100 \%$ if the phase constant of the leaky-wave meets exactly the free space wavenumber (ordinary end-fire condition) and the aperture length is adjusted with regard to the attenuation constant of the leaky-wave from the modal analysis. For a given aperture length, a procedure is outlined to adjust the attenuation constant in several steps at the desired ordinary end-fire frequency to reach maximum gain and efficiency.
\end{abstract}

\section{Introduction and first design analysis}

Figure 1a, shows the configuration with infinite length and filled rectangular waveguide used for the modal analysis in the space domain (Liu and Jackson, 2011). This analysis searches for the complex propagation wavenumber $k_{z}=$ $\beta-j \alpha$ which sets the corresponding transcendental equation to zero (Eq. 7 in Liu and Jackson, 2011). For this search, we use the downhill-method (Bach, 1969), leading to convergent results typically with around 50 steps. The first design is based on the following values related to a wavelength $\lambda_{\mathrm{o}, \mathrm{e}}$, which corresponds to the free space wavenumber at the frequency of ordinary end-fire operation $f_{\mathrm{o}}$, e, see text below. Due to non-disclosure reasons, the exact frequencies (in the $K_{u}$-band) and design parameters must not be specified: slot length $L=0.16 \lambda_{\mathrm{o}}$, e slot width $W=0.025 \lambda_{\mathrm{o}, \mathrm{e}}$, periodicity $p=0.15 \lambda_{\mathrm{o}, \mathrm{e}}, b=0.44 \lambda_{\mathrm{o}, \mathrm{e}}, a=w_{\mathrm{eff}}=0.3 \lambda_{\mathrm{o}}$ e and the dielectric filling $\varepsilon_{r}=3.5$. In Fig. $2 b$, the modal analysis of the propagation constant $\beta / k_{0}$ is given. For frequencies larger than $f_{\mathrm{o}, \mathrm{e}}$, the leaky-wave (blue) and the proper waveguide mode (green) have a $\beta / k_{0}>1$. According to O'Connor and Jackson (2011), the (modified) HansenWoodyard condition for highest directivity and gain with an aperture length of $\approx 20 \lambda_{0, \text { e }}$ should be fulfilled for $\beta / k_{0} \approx$ 1.023 reached around $1.0076 f_{\mathrm{o}, \mathrm{e}}=f_{H . W}$., the frequency where the Hansen-Woodyard condition is fulfilled. According to Fig. $2 b$, the attenuation constant with dielectric losses reaches $\alpha / k_{0}=0.0161$ at $f_{H . W}$. (blue curve at red bar). But if the structure is analyzed with a finite length as in Fig. 1b, the attenuation drops down for frequencies beyond the ordinary end-fire operation.

This can be recognized by the behaviour of $\left|S_{12}\right|$ reaching a minimum exactly at the ordinary end-fire frequency with $-9.21 \mathrm{~dB}$ and a radiation efficiency of $e_{r}=88 \%$ and then in- 

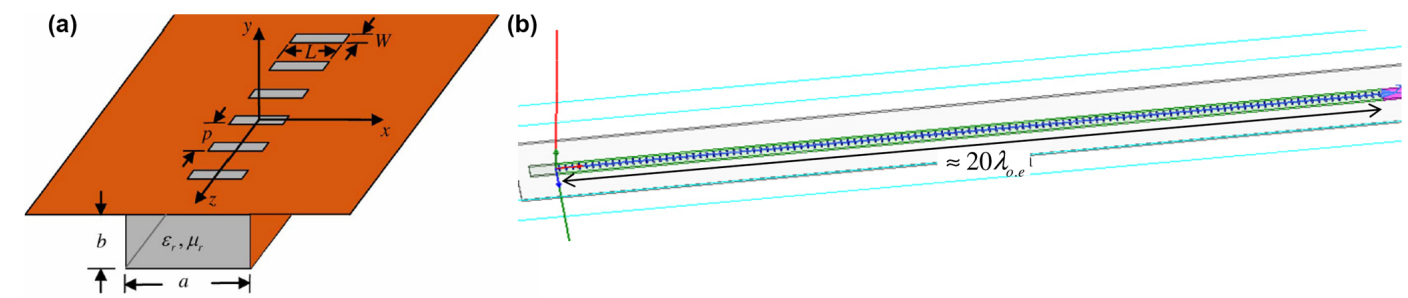

Figure 1. (a) Configuration for the modal analysis. (b) Model in HFSS for analyis of antennas with finite length.
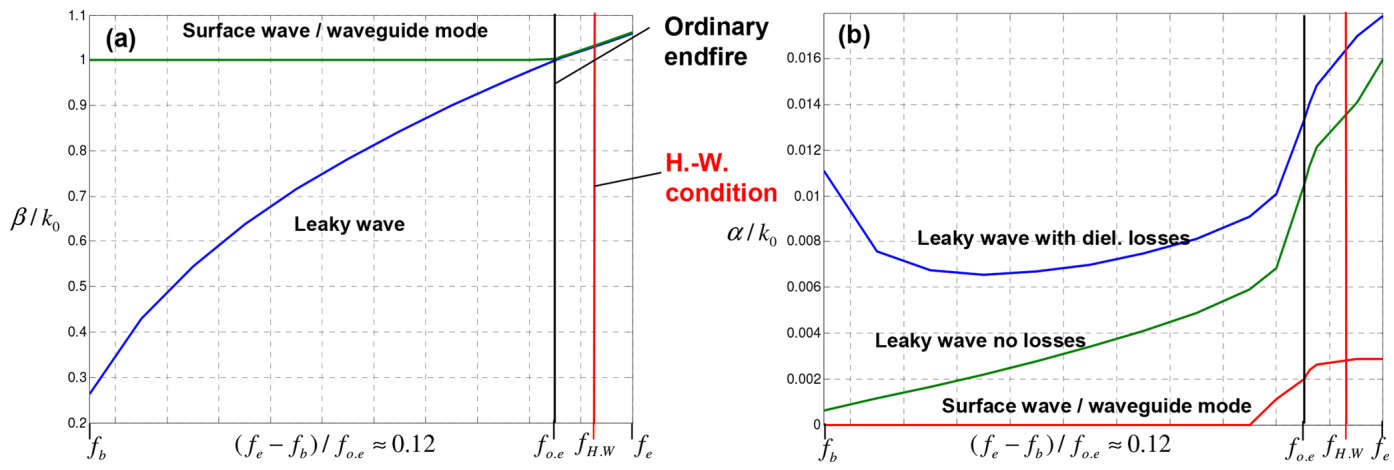

Figure 2. Modal analysis results of the structure in Fig. 1a.
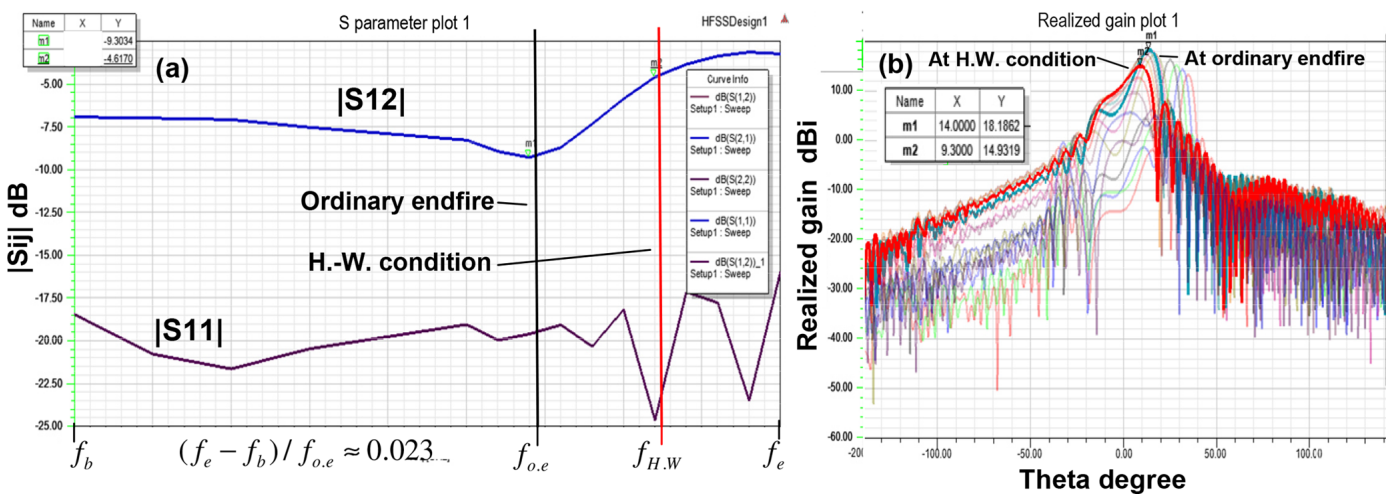

Figure 3. Analysis of the structure in Fig. 1b with HFSS.

creases strongly to $-4.73 \mathrm{~dB}\left(e_{r}=66 \%\right)$ where the modified Hansen-Woodyard condition should be fulfilled (see Fig. 3b).

This is due to the fact, that the leaky mode becomes unphysical beyond end-fire and the proper waveguide mode becomes dominant with a low attenuation only due to dielectric and metallic losses, thus $\left|S_{12}\right|$ becomes larger.

\section{Gain improvement by maximizing the efficiency}

This can also be observed in Fig. 2b, where the red curve shows only a small increase somewhat before $f_{\mathrm{o}, \mathrm{e}}$. Figure $3 \mathrm{~b}$ shows the radiation pattern at ordinary end-fire (blue) with a maximum gain of $18.19 \mathrm{dBi}$, whereas the gain curve at the Hansen-Woodyard condition shows only a maximum of $14.93 \mathrm{dBi}$ mainly due to the lower radiation efficiency. Ac- cording to Liu and Jackson (2012), the contribution of the leaky-wave drops down to about $10 \%$ at ordinary end-fire, whereas the surface-wave mode may show a maximum contribution around this frequency.

Obviously the leaky-wave is transformed into the surfacewave over the aperture length in such a way that the attenuation constant for the leaky-wave derived from the modal analysis is still valid for the radiating mechanism of both leaky and surface-wave mode up to the frequency of ordinary end-fire. Since the excitation of the surface wave drops down beyond the ordinary end-fire frequency, the efficiency of $86 \%$ at the modified Hansen-Woodyard condition for gain maximization as proposed in O'Connor and Jackson (2010) cannot be reached. Therefore we propose as follows a gain improvement at the ordinary end-fire frequency by a proce- 

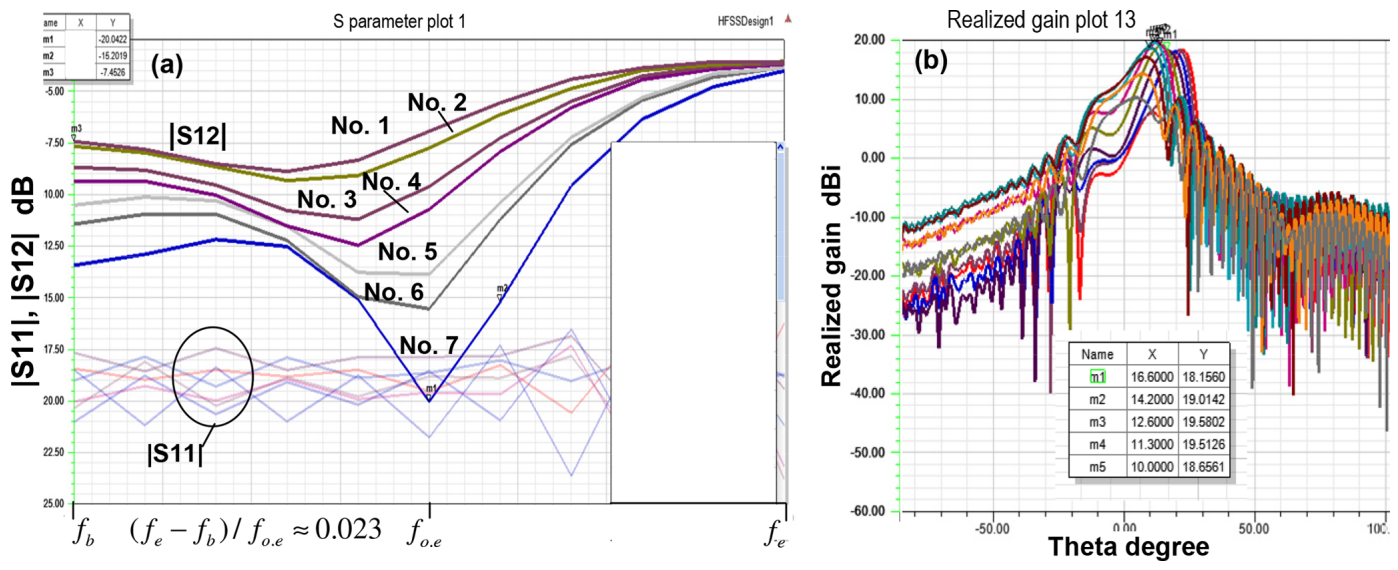

Figure 4. (a) S-parameters of the structure Fig. 1b with the values of Table 1. (b) Gain pattern of the structure No. 7.
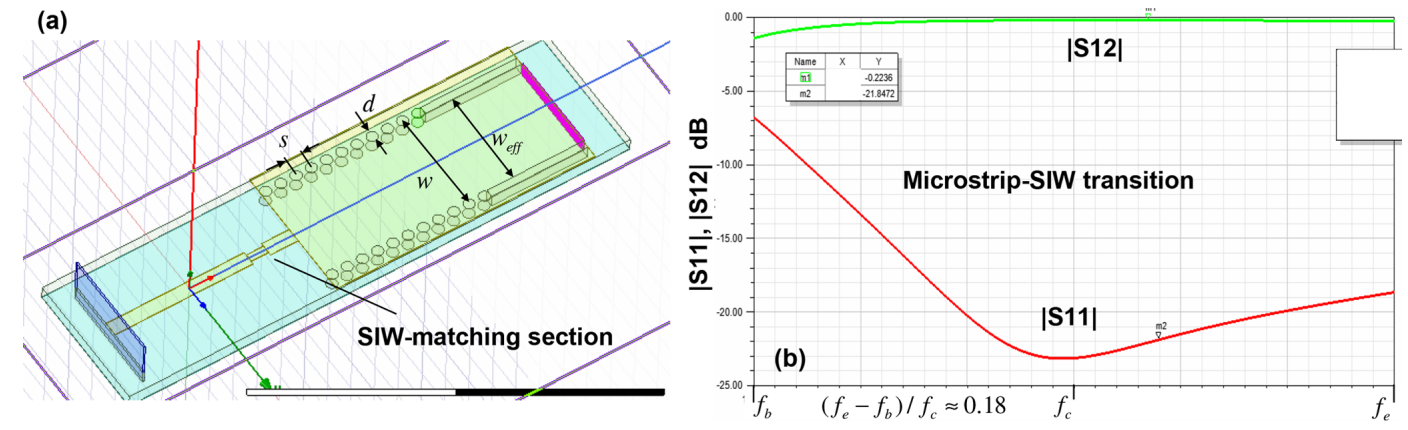

Figure 5. Microstrip-SIW transition.
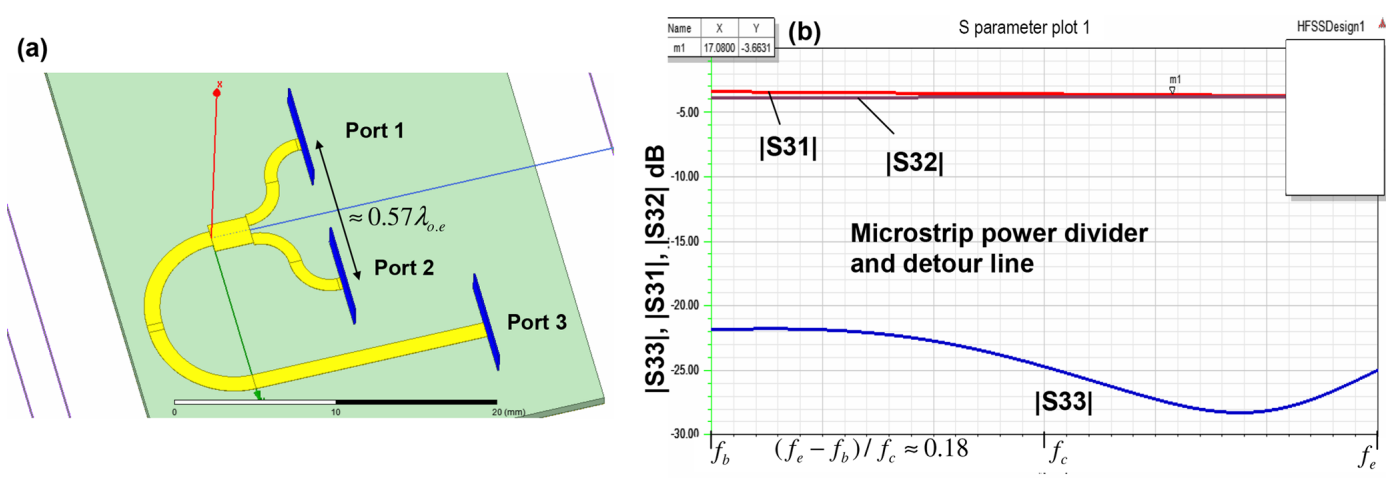

Figure 6. Microstrip power divider with detour line.

dure of step-wise increasing the attenuation constant to maximize the efficiency derived by

$\varepsilon_{r}=1-\left|S_{12}\right|^{2}$

and to maximize the gain at ordinary end-fire.

The attenuation constant can be increased by enlarging the slot length and/or the slot width and/or decreasing the slot periodicity. But with fixed width of the waveguide, also the values of $\beta / k_{0}$ are increasing. This can be compensated by decreasing the width of the waveguide. A good estimation for the modification of the width by $\Delta w_{\text {eff }}$ is given by (similar as in Liu and Jackson, 2014)

$$
\Delta w_{\mathrm{eff}} \approx \frac{\pi}{\sqrt{k_{0}^{2} \varepsilon_{r}-\beta^{2}\left(L, W, w_{\mathrm{eff}}, p\right)}}-\frac{\pi}{\sqrt{k_{0}^{2} \varepsilon_{r}-\beta_{\mathrm{desired}}^{2}}}
$$

where $\beta\left(L, W, w_{\text {eff }}, p\right)$ is the propagation constant with the current $w_{\text {eff }}$ and $\beta_{\text {desired }}$ is the desired propagation constant for the given $L, W, p$ of the slots. However, in most cases it is faster to use a linear interpolation to find the exact $w_{\text {eff }}$ : If $\beta$ is too large, $w_{\text {eff }}$ is slightly decreased and from the new 


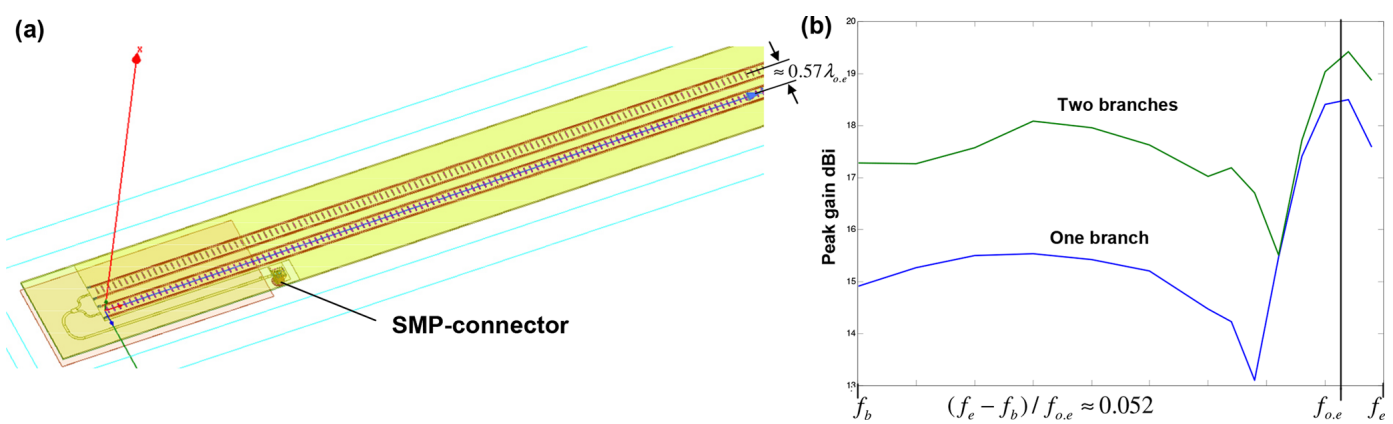

Figure 7. (a) Structure with two antenna branches. (b) Comparison of peak gain of structures with two and one antenna branches.

Table 1. Stepwise increasing of the attenuation constant at ordinary end-fire.

\begin{tabular}{lrrrrrr}
\hline No. & $L / \lambda_{\mathrm{o}, \mathrm{e}}$ & $p / \lambda_{\mathrm{o}, \mathrm{e}}$ & $w_{\text {eff }} / \lambda_{\mathrm{o}, \mathrm{e}}$ & $\beta / k_{0}$ & $\alpha / k_{0}$ & $g_{\max }(\mathrm{dBi})$ with HFSS \\
\hline 1 & 0.173 & 0.242 & 0.3033 & 1.0004 & $1.013 \times 10^{-2}$ & $18.28\left(0.995 f_{\mathrm{o}, \mathrm{e}}\right)$ \\
2 & 0.173 & 0.230 & 0.3027 & 0.999 & $1.056 \times 10^{-2}$ & $18.43\left(0.995 f_{\mathrm{o}, \mathrm{e}}\right)$ \\
3 & 0.173 & 0.202 & 0.3016 & 1.0007 & $1.29 \times 10^{-2}$ & $18.94\left(0.997 f_{\mathrm{o}, \mathrm{e}}\right)$ \\
4 & 0.173 & 0.190 & 0.3010 & 1.0002 & $1.402 \times 10^{-2}$ & $19.2\left(0.997 f_{\mathrm{o}, \mathrm{e}}\right)$ \\
5 & 0.173 & 0.173 & 0.2998 & 0.999 & $1.563 \times 10^{-2}$ & $19.4\left(1.000 f_{\mathrm{o}, \mathrm{e}}\right)$ \\
6 & 0.173 & 0.161 & 0.2993 & 1.0009 & $1.76 \times 10^{-2}$ & $19.52\left(1.000 f_{\mathrm{o}, \mathrm{e}}\right)$ \\
7 & 0.173 & 0.145 & 0.2981 & 1.0005 & $2.026 \times 10^{-2}$ & $19.58\left(1.000 f_{\mathrm{o}, \mathrm{e}}\right)$ \\
\hline
\end{tabular}

value for $\beta$, the exact $w_{\text {eff }}$ can be often determined with one interpolation step. We have now implemented this method as an iterative procedure in our modal analysis program showing typically a very good convergence in 2 to 4 steps with a maximum deviation for $\beta_{\text {desired }} / k_{0}$ of 0.001 .

In Table 1 , the $\alpha / k_{0}$ with the parameter set No. 1 is doubled in 6 steps only by reduction of the periodicity $p(L=$ $0.173 \lambda_{\mathrm{o}, \mathrm{e}}$, and $W=0.0254 \lambda_{\mathrm{o}, \mathrm{e}}$ remain constant), keeping the $\beta / k_{0}$ at the ordinary end-fire value for the constant frequency $f_{\mathrm{o}, \mathrm{e}}$ using the modal analysis and the interpolation sketched above. The values for the maximum gain are derived with HFSS with the aperture length of the structure in Fig. 1b. Further results of the simulations with HFSS are given in Fig. 4. The minima of $\left|S_{12}\right|$ become monotonously smaller, for the parameter sets No. 1 to No. 4 we have still a small frequency offset, but from parameter set No. 5 the frequency with maximum attenuation at the desired frequency $f_{\mathrm{o}, \mathrm{e}}$ is also met by the HFSS-simulations. With Eq. (1) we get an efficiency of $97 \%$ for No. 6 and $99 \%$ for No. 7, thus a further increase of the attenuation constant is not meaningful and would lead to a gain reduction. Figure $4 \mathrm{~b}$ shows the gain pattern of No. 7 for different frequencies .

The gain is, depending on frequency, up to $1.4 \mathrm{dBi}$ higher for No. 7 compared with the parameter set No. 1. A radiation exactly in end-fire direction is not possible due to diffraction effects of the finite groundplanes.

Since the antennas are fabricated in SIW-technology, we still need a SI-waveguide with width $w$ and the same propagation constant as the rectangular waveguide with width $w_{\text {eff }}=a$ (see also Fig. 5a). In many cases the formula (e.g. Kumar and Jadhav, 2012)

$w=w_{\text {eff }}+d^{2} /(0.95 \mathrm{~s})$

Equation (3) is not correct enough. With $d=0.6 \mathrm{~mm}$ and $s=$ $0.8815 \mathrm{~mm}$ we get $w=0.3229 \lambda_{\mathrm{o}}$, e with Eq. (3) what leads to a minimum of $\left|S_{12}\right|$ around $100 \mathrm{MHz}$ higher than the desired $f_{\mathrm{o}, \mathrm{e}}$. But if we take the formula

$w=w_{\mathrm{eq}}+s \cdot\left(0.766 e^{0.4482 d / s}-1.176 e^{-1.214 d / s}\right)$,

derived in Kordiboroujeni and Bornemann (2013), we get $w=0.3248 \lambda_{\mathrm{o}}$, e slightly higher, the minimum is shifted to the desired $f_{\mathrm{o}}$, e again and we get the same gain behaviour as with a rectangular waveguide.

For the practical implementation we need also a microstrip-SIW transition, which is sketched in Fig. 5, showing a good matching behavior with low losses and a bandwidth of about $18 \%$.

\section{Antennas with two branches}

To reach a higher gain also for frequencies below end-fire, we have also examined structures with two antenna branches. In this context we need a power divider with low losses. A suitable microstrip divider with additional detour line is outlined in Fig. 6 with the input port 3. We have used rounded bends to minimize the parasitic radiation leading to losses of only about $0.6 \mathrm{~dB}$ (Weisshaar and Tripathi, 1990). A power 
divider with mitered bends could be optimized for a good matching behaviour, but the losses amount to about $1.5 \mathrm{~dB}$ mainly due to the parasitic radiation of the mitered bends. A structure with two antenna branches with a center distance of $10 \mathrm{~mm}$ is given in Fig. 7a.

Figure $7 \mathrm{~b}$ shows the peak gain comparison with one and two branches. Due to mechanical constraints, the SMPconnector must be located alongside the branches thus the additional detour line is needed. The SMP-connector is linked to the microstrip line with an additional matching section .

The maximum gain of the structure with one branch

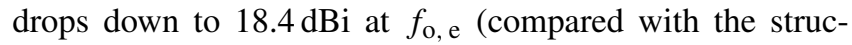
ture No. 7 with $19.58 \mathrm{dBi}$ ) due to the additional detour line, microstrip-SIW transition and the SMP-connector. But with two branches we get a maximum gain of $19.42 \mathrm{dBi}$ and for frequencies below end-fire, the peak gain is typically $3 \mathrm{~dB}$ higher compared with the structure with only one branch.

\section{Conclusions}

Leaky-wave antennas in SIW-technology with transverse slots are a good candidate, if a frequency scanning capability up to end-fire and a cheap fabrication is desired. To increase the directivity and gain at end-fire operation, the (modified) Hansen-Woodyard condition may be applied. To fulfill this condition, the propagation constant of the radiating mode must be slightly higher than the free space wavenumber. However, in this slow wave region the leaky-wave becomes unphysical and also the excitation of the surface wave mode drops down strongly beyond the ordinary end-fire operation. But it could be found, that the leaky-wave is transformed into the surface wave at the frequency of ordinary end-fire and that for the radiation loss computation along the aperture still the attenuation constant of the leaky-wave can be used which is derived from a modal analysis based on rectangular waveguides. With this modal analysis, we have outlined a procedure to stepwise increase the attenuation constant e.g. by decreasing the periodicity of the slots and decreasing the width of the waveguide to get a radiation efficiency of about $99 \%$ adjusted to the length of the aperture. After a careful transformation of the rectangular waveguide into a SIW together with an efficient microstrip-SIW transition, a cheap fabrication is possible. With the use of power dividers with low losses, structures with two antenna branches can be designed leading to a gain improvement also for frequencies below end-fire operation.

Data availability. There are no underlying research data for the presented work due to non-disclosure reasons. The results can be reproduced with the design methods and equations given in the paper.
Competing interests. The authors declare that they have no conflict of interest.

Special issue statement. This article is part of the special issue "Kleinheubacher Berichte 2018". It is a result of the Kleinheubacher Tagung 2018, Miltenberg, Germany, 24-26 September 2018 .

Review statement. This paper was edited by Thomas Eibert and reviewed by three anonymous referees.

\section{References}

Bach, H.: On the downhill method, Commun. ACM, 12, 675-677, 1969.

Kordiboroujeni, Z. and Bornemann, J.: Designing the Width of Substrate Integrated Waveguide Structures, IEEE Microw. Wirel. Co., 23, 518-520, 2013.

Kumar, H., Jadhav, R., and Ranade, S.: A Review on Substrate Integrated Waveguide and its Microstrip Interconnect, IOSR Journal of Electronics and Communication Eng. (IOSR-JECE), 3, 36-40, 2012.

Liu, J. L., Jackson, D. R., and Long. Y.: Modal Analysis of Dielectric-Filled Rectangular Waveguide With Transverse Slots, IEEE Trans. Antennas Prop., 59, 3194-3203, 2011.

Liu, J. L., Jackson, D. R., and Long, Y.: Substrate Integrated Waveguide (SIW) Leaky-Wave Antenna With Transverse Slots, IEEE Trans. Antennas Prop., 60, 20-29, 2012.

Liu, J. L., Jackson, D. R., Li, Y., Zhang, C., and Long, Y.: Investigations of SIW Leaky-Wave Antenna for End-fire-Radiation With Narrow Beam and Sidelobe Suppression, IEEE Trans. Antennas Prop., 62, 4489-4497, 2014.

O'Connor, E. M., Jackson, D. R., and Long, S. A.: Extension of the Hansen-Woodyard Condition for End-fire Leaky-Wave Antennas, IEEE Antenn. Wirel. Pr., 9, 1201-2104, 2010.

Weisshaar, A. and Tripathi, V. K.: Perturbation Analysis and Modeling of Curved Microstrip Bends, IEEE Trans. Microwave Theory Techn., 38, 1449-1454, 1990. 